\title{
Computer simulation of FIM images - the convex hull model
}

\author{
A. Łukaszewski ${ }^{\mathrm{a}}$, A. Szczepkowicz ${ }^{\mathrm{b}, *}$ \\ ${ }^{a}$ Institute of Computer Science, University of Wroctaw Przesmyckiego 20, 51-151 Wroctaw, Poland \\ ${ }^{\mathrm{b}}$ Institute of Experimental Physics, University of Wroctaw, Maxa Borna 9, 50-204 Wroctaw, Poland
}

\begin{abstract}
A new simulation method of field emission ion images is proposed. The method can be applied to any crystal shape, size and atomic structure (possibly with point defects and dislocations), without any trial-and-error adjustment of parameters. The algorithm is illustrated by three simple examples: spherical crystals of perfect FCC, BCC and HCP structures. (C) 1999 Elsevier Science Ltd. All rights reserved.
\end{abstract}

\section{Introduction}

The image observed in a field ion microscope (FIM) shows a complex structure of many crystal faces. In order to understand various aspects of this image, it is often convenient to compare it with a theoretical model of the specimen. In the early days of field ion microscopy several investigators have used ball models for this purpose $[1,2]$. In 1962 Moore proposed the first computer model [3], which became known as the thin shell model, and which is nowadays the most widely used, although other methods of simulation have also been developed. We will not discuss the various existing models in detail, as there exists a comprehensive review article on this subject by Suvorov et al. [4].

\section{General scheme of constructing a model of a FIM image}

Computer simulation of FIM image consists roughly of the following steps:

1. Determination of atom positions in a crystal lattice.

2. Determination of crystal shape.

3. Determination of the atoms that are visible.

4. Projection of the visible part of crystal surface onto the "screen".

5. Drawing of the image spots.

\footnotetext{
*Corresponding author. E-mail: asz@ifd.uni.wroc.pl
}

In the first step an assumption must be made about the atomic structure of the sample. The simplest and often considered case is a perfect crystal, when the atoms form a Bravais lattice, or a superposition of Bravais lattices. Some algebraic methods useful for computing atom positions of perfect cube-based lattices are described in the context of FIM simulations by Eaton and Lee [5]. Simulation of various lattice defects requires some more computational effort [4].

The second step is more difficult. Real samples are shaped by field evaporation, or by surface diffusion during annealing, or by a combination of both. Consequently, it would be best to perform a dynamical simulation of these processes in order to determine a realistic sample shape. However, such simulations have not yet been applied to ion image modeling. Instead, most researchers assume a spherical sample shape as a model.

Steps 1 and 2 allow to calculate the positions of all the atoms in the sample. Obviously only a small fraction of these atoms gives rise to spots in the ion image.

In the third step one must choose an imaging criterion-a method to determine which of the crystal atoms are actually visible in the image. So far, there were two approaches:

(a) The thin shell model. In this model, the imaging criterion is the distance of the atom from an ideal sphere which represents the surface. All atoms of the crystal lying within some critical distance $d$ from the surface are plotted in the simulated image.

(b) The bond models. This type of criteria is based on the bonding schemes of atoms. For each atom, it is 
necessary to count the number of nearest neighbors $n_{1}$, second nearest neighbors $n_{2}$, etc. Only if an atom has the "correct", sufficiently small number of neighbours of every type, it is considered an image point. The maximum allowed values of $n_{1}, n_{2}, \ldots$ depend on the author. One of the best bond models is described by Vinogradov et al. [6,7].

The fourth step of simulation consists in finding a projection of the crystal surface onto the screen plane. Most authors use orthographic or stereographic projections. However, it is best to calculate the actual electric field between the two electrodes: the screen and the sample. Then the real ion trajectories can be calculated, and their intersection with the screen could be taken as the spot positions. Such calculations have been reported [8,9], but only for simple (e.g. paraboloidal) emitter shapes, which are only a rough approximation of a field evaporated or a thermally equilibrated crystal.

In the fifth, final step of simulation one has to choose a graphical representation of the atomic spots. This issue has not been given much attention in the past because of the limitations of the printing devices. An atom was often represented by a smallest dot that a particular device could draw. With today's high resolution devices one can visualize a spot as a circle of arbitrary diameter and brightness distribution. However, these parameters are a complicated function of tip temperature, field strength, and imaging gas used and computation of the proper shading model requires further investigation.

\section{The convex hull imaging criterion}

In this paper, we concentrate only on the third step of simulation - we develop a new imaging criterion. We do not apply any new methods for steps 1, 2, 4 and 5 .

The imaging criteria described in the previous section have the following shortcomings:

(a) The thin shell model contains a problematic parameter $d$ - the shell thickness. The physical meaning of this parameter is obscure. The parameter has to be adjusted by trial and error to match the actual number of spots in a real ion photograph. Furthermore, it is necessary to choose different values of $d$ for different tip radii. The thin shell criterion can be extended to other simple emitter shapes, for example elliptic or parabolic, but it would not work with more realistic shapes.

(b) The bond models are more justified physically, and they can be applied to any crystal shape, but they assume a perfect crystal lattice, with no interstitial atoms or dislocations.

We will now describe a simple imaging criterion which is free from the shortcomings described above. The idea behind this criterion is that a "visible" atom must sit in a relatively open position, so that it can be accessed by the image gas ions. This is a purely geometrical property. We do not take into account other factors which affect the visibility, such as the electric field intensity above the atom.

Imagine a plane passing through the center of an atom. This plane divides the three-dimensional space into two half-spaces. If this plane can be placed so that one of the half-spaces is empty, i.e. all other atomic centers lie either in the other half-space or on the plane, then we assume that the atom is visible.

An alternative, more concise definition of this criterion can be given with the use of the following widely used mathematical notion which is central in computational geometry: The convex hull of a set of points is the smallest convex set containing all given points. Now we can state our imaging criterion as follows:

An atom is imaged if and only if it lies on the boundary of the convex hull of the crystal.

In this definition a crystal is understood as the set of atomic centers.

Fig. 1 illustrates the above definitions in two dimensions. The polygon outlined by solid line is the convex hull of a two-dimensional crystal. The atoms which satisfy the criterion are drawn in black. In three dimensions, a convex hull of a finite set of atoms is a polyhedron. The faces of the polyhedron are the crystal faces.

Figs. 2-4 show simulated ion images which were generated with the use of the convex hull model. The following assumptions were made:

1. The crystal structure is perfect (no interstitials or dislocations).

2. The crystal lattice is truncated by a sphere. The radius of the sphere is equal to 150 lattice constants.

3. Only the atoms satisfying the convex hull criterion are imaged.

4. The projection is stereographic. Only the surface within the azimuthal angle $\theta \leqslant 60^{\circ}$ is projected.

5. The image spots are solid circles. Their radius is equal to the nearest neighbor distance of the crystal lattice.

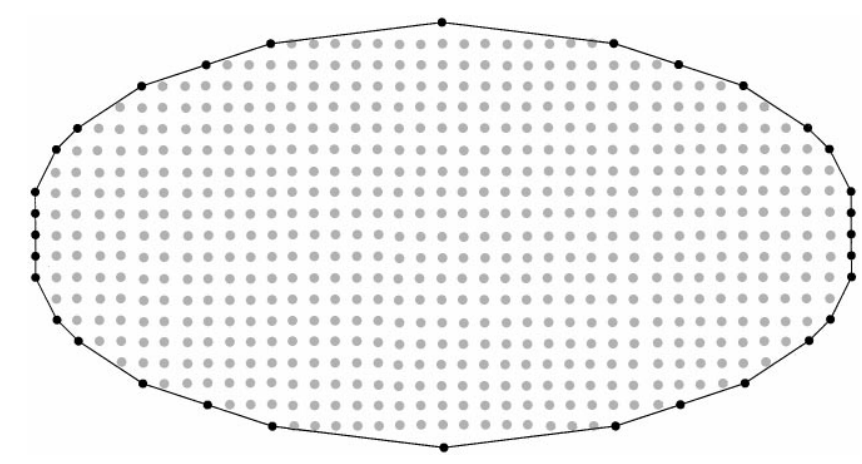

Fig. 1. The convex hull of a two-dimensional set of points. 


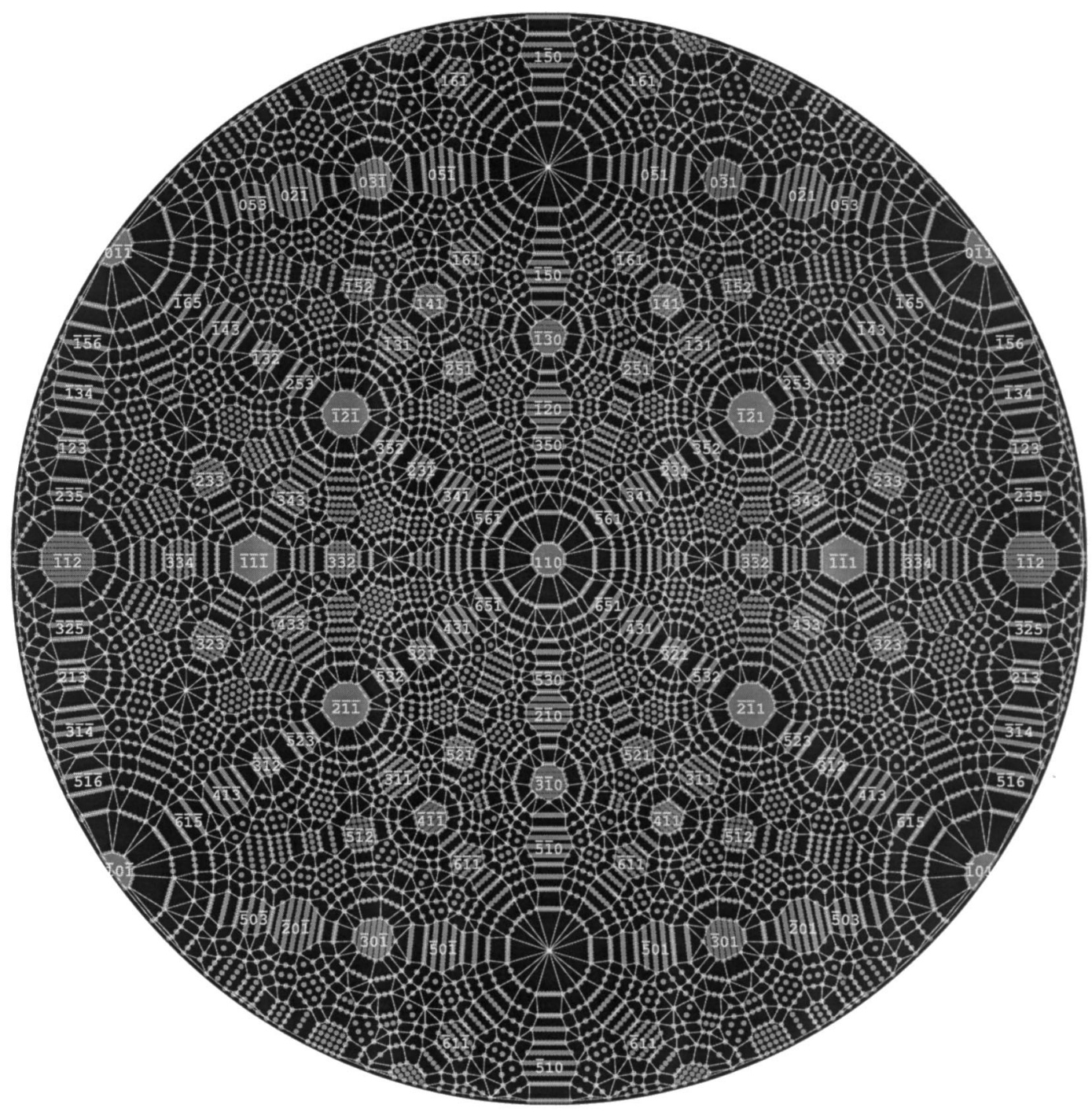

Fig. 2. The atoms lying on the convex hull of a spherical BCC crystal.

This roughly corresponds to the liquid nitrogen imaging temperature.

Figs. 2-4 include two additional features which may be of interest to a FIM researcher. The first one is that the crystallographic indices of the main crystal faces are given (only the faces consisting of at least 30 atoms are indexed). The second one is that all convex hull edges are shown, or, in other words, all crystal faces are outlined.

As can be observed from Figs. 2-4, the convex hull model gives realistic results except for the densely packed crystal faces, where, in contradiction with the real images, all atoms of the face are visualized. For this reason the total number of visualized atoms is greater than in the real images. For example, in Fig. 2, there are 1080 atoms in the crystallographic triangle 100-110-111, compared to experimental value of about 700 atomic spots for field evaporated end forms [10]. Apart from this effect, the similarity of the generated image to the real-ion photographs seems to be limited only by the assumption of a simple, spherical shape. One may expect much better results of the convex hull model applied to a field equilibrium shape of the crystal.

\section{Computational issues}

Although the mathematical formulation of the convex hull criterion is very simple, the actual calculation of a convex hull in three-dimensional space for huge numbers of points is quite difficult. One of the first algorithms was given in 1970 by Chand and Kapur [11]. Further advances in this field have been done during late 1970s and 1980s when several more efficient algorithms were proposed, with running time of the order $n \log n(n=$ number of points). One of the randomized algorithms has been 


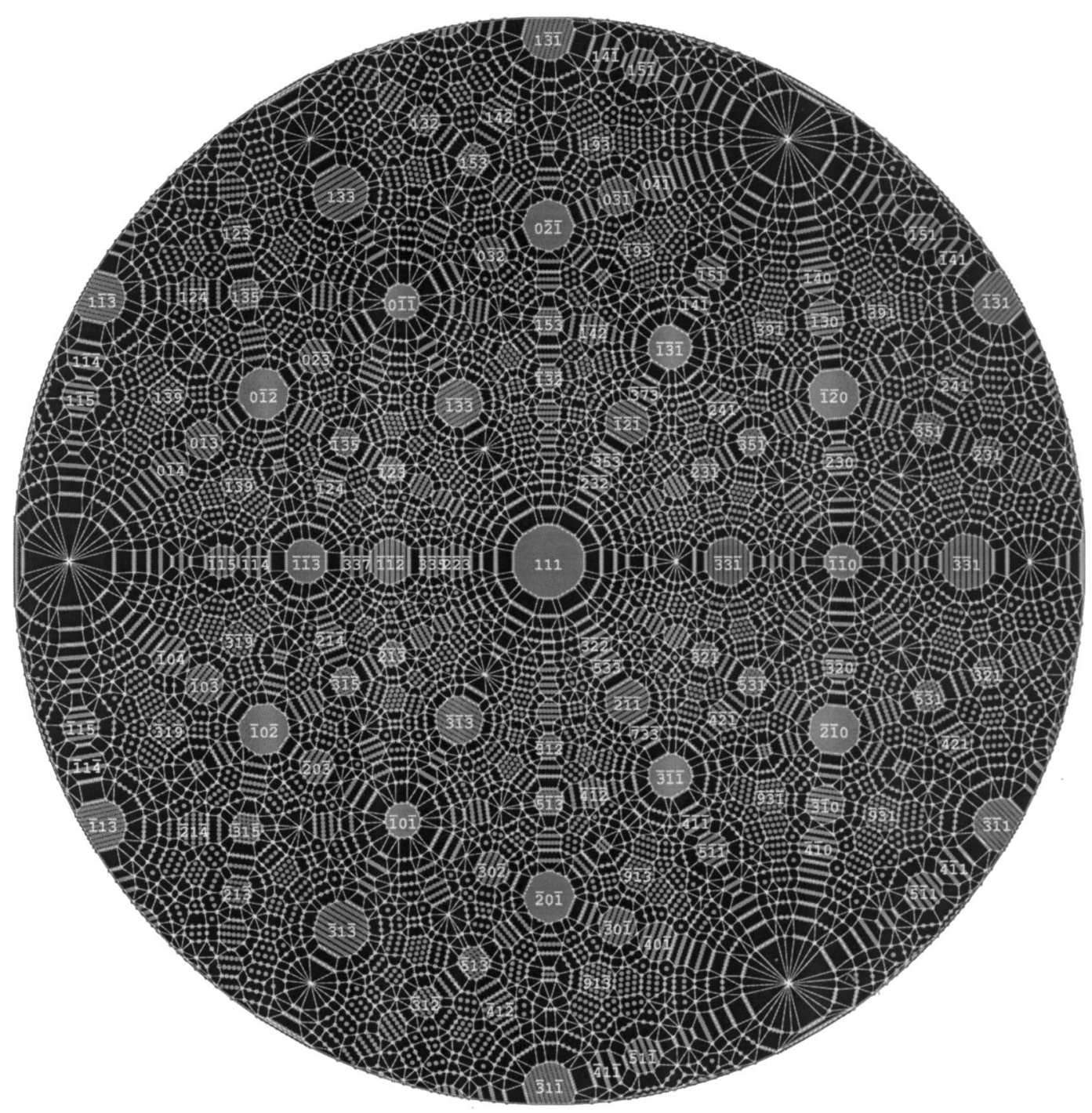

Fig. 3. The atoms lying on the convex hull of a spherical FCC crystal.

found by Clarkson and Shor [12] (this algorithm is also described by de Berg et al. [13] ). In the computer program developed for this work we used similar randomized algorithm which is implemented in the LEDA algorithm library [14].

The computation of a convex hull is time and memory consuming. For the algorithm used, both computing time and memory usage are proportional to $n \log n$, where $n$ is the number of points. For this reason it is important to perform careful initial selection of the points to be considered by the algorithm. Points which can easily be shown to lie inside the hull should be removed. Such selection obviously does not alter the convex hull of the crystal. In the examples calculated in this work, it was sufficient to consider only the atoms lying within lattice constant of the sphere. After such selection, the computations take minutes on a current PC or workstation.

\section{Conclusion}

The convex hull model presented in this work is a simple geometric imaging criterion. Its underlying physical assumption is clear and can be stated as follows: on all facets, the atoms imaged are the ones of the first lattice plane. Although this is only an approximation of experimental facts, the images generated are useful "crystallographic maps" which can aid a FIM researcher in understanding the crystallography of the real images. The greatest difficulty that hinders the comparison of theory and experiment is that a sphere is a poor approximation of field evaporated crystal surface. So the still remaining challenge is to simulate the process of formation of a field equilibrium crystal shape. Once such crystal form is calculated, the convex hull criterion can easily be applied to give much better agreement with experiment. 


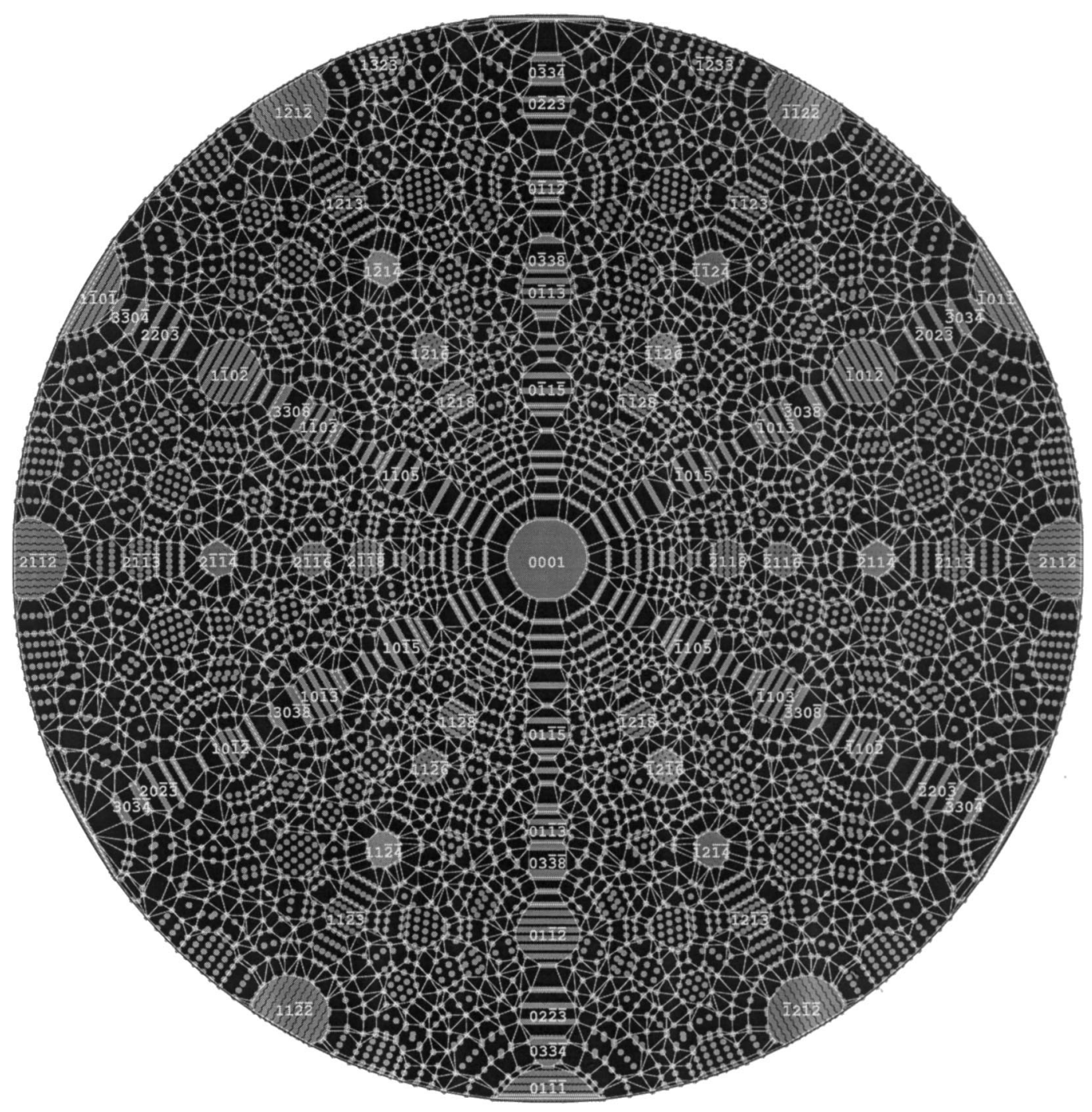

Fig. 4. The atoms lying on the convex hull of a spherical HCP crystal.

\section{Acknowledgements}

This work was supported by the Maria SkłodowskaCurie Foundation, Grant MEN/DOE-96-277.

\section{References}

[1] Müller, EW. Adv Electron Electron Phys 1960;13:83.

[2] Drechsler M, Wolf P. IV Int Kongress Elektronenmikroskopie, Berlin, 1958. Berlin: Springer, 1960;1:835.

[3] Moore AJW. J Phys Chem Solids 1962;23:907.

[4] Suvorov AL, Razinkova TL, Sokolov AG. Phys Stat Sol A 1980;61:11.
[5] Eaton HC, Lee L. J Appl Phys 1982;53(2):988.

[6] Vinogradov VV, Borisov VT, Potapow LP, Kristallografiya 1974;19:138

[7] Vinogradov VV. Kristallografiya 1974;19:1229.

[8] Smith R, Walls JM. J Phys D 1978;11:409.

[9] Gipson, GS. J Appl Phys 1980;51(7):3884.

[10] Moore AJW, Ranganathan S. Phil Mag 1967;16:723.

[11] Chand DR, Kapur SS. J ACM 1970;17:78.

[12] Clarkson KL, Shor PW. Discrete Comput Geom 1989;4:387.

[13] de Berg M, van Kreveld M, Overmars M, Schwarzkopf O. Computational geometry Berlin: Springer, 1997.

[14] http: //www.mpi-sb.mpg.de/LEDA, Max-Planck-Institut für Informatik, Saarbrücken, Germany. 